\title{
ZUR PRODUKTION UND REZEPTION DES CARTOONS IN "ZEITMAGAZIN"
}

In diesem Beitrag wird versucht, die Gesetzmässigkeiten der Textsorte Cartoon, wie er in "Zeitmagazin" vorkommt, zu beschreiben und ihre Bedeutung für die Textrezeption zu erklären. Im ersten Teil wird kurz auf die Textsorte Cartoon als Gegenstand des vorliegenden Beitrags eingegangen, im zweiten wird die Rolle des Textproduzenten bei der Textkonstitution und die des Rezipienten beim Textverstehen berührt.

Nach Eco (1991: 24) gehören Comic strips zu visuellen Phänomenen in den Massenkommunikationen. Dies sei das Feld, wo die Semiotik immer mehr an Geltung gewinne.

In den meisten deutsch-deutschen Wörterbüchern sind folgende Definitionen des Begriffs Cartoon zu finden:

- gezeichnete oder gemalte, häufig satirische Geschichte in Bildern (Comic strip)

- Karikatur, satirische Zeichnung

Im Brockhaus/Wahrig (1980) ist der oben in Klammern erwähnte Comic strip definiert als in Streifen gezeichnete Bilderfolge zur Darstellung von meist komischen, aber auch abenteuerlichen, utopischen u.a. Handlungsabläufen, wobei die Erzählsituation durch knappe, den Handelnden in Form von Sprechblasen in den Mund gelegte Sätze u. durch eingeschobene Texte erläutert wird.

Weber (1984: 339) definiert Comic strips folgenderweise: "...comics consist of images representing animated beings or things, and of words representing the verbal behaviour of these beings."

In diesem Beitrag werden nur diejenigen Cartoons analysiert, die ausschließlich aus einem Bild und einem Text bestehen. Der kinematographische Teil des Comic strips, d.h. in Streifen gezeichnete Bilderfolge, ist somit nicht Gegenstand dieser Untersuchung. Unter Text ist hier entweder als ein relativ kurzer Übertitel oder Untertitel zu verstehen, oder ein relativ langer Text, der sich ausschließlich unter dem Bild befindet.

Die Tatsache, dass einem längeren Text immer der bildliche Teil des Cartoons vorangeht, ist auf die psychologische Wirkung zurückzuführen, dass die 
Aufmerksamkeit des Lesers/Zusehers zuerst auf Bewegtes, Dynamisches, Farbiges, Buntes gelenkt wird, erst dann auf Statisches, Monotones, Schwarzweißes, Langweiligeres (S. Cartoon 9, 10, 11, 12, 13, 14). Wenn Texte in Sprechblase(n) oder in ähnlicher Form realisiert sind, dann entfällt der Übertitel bzw. Untertitel in den meisten Fällen (S. Cartoon 1, 2, 3).

Oft stellt sich die Frage, ob Comic strips bzw. cartoons überhaupt als Texte angesehen werden können. Hier folgen zwei Erklärungsversuche, aus denen hervorgeht, dass es doch angebracht ist, sie als Texte zu betrachten:

"From the beginning of text linguistics it has been agreed on by many workers in this field that methods of textual research can be applied to other types of 'semiotic texts', such as pictures, stage plays, movies etc., as these media are based on typical connecting elements which are relevant to the attitudes and intentions of authors/artists and recipients in specific situations. Comics, too, can be treated as special types of 'texts' with a rich repertory of connecting devices. Some of these can be regarded as adopted from other media, e.g. stage play, writing, illustration, (silent) movie etc. For this reason, much of what can be said about connectedness in texts and discourses, paintings and photographs, stage plays and motion pictures, applies to comics and vice versa." (Weber, 1984: 337)

"Comics können...als Texte angesehen werden, jedenfalls wenn die Bilder mit Sprachmaterial (in Form von Sprechblasen usw.) verbunden sind und gegenseitige Verweisbeziehungen bestehen." (Vater, 1992: 18)

Cartoon als Textsorte fällt somit in die Gruppe gemischter Texte, wobei die Dominanz von sprachlichen oder außersprachlichen Elementen nicht einfach festzustellen ist. Das Bild und der Text scheinen sich gleichwertig zu unterstützen, d.h., der Informationswert ist auf beiden Textteilen gleichmäßig verteilt. Es gibt ganz wenige Fälle, wo der Text fehlt (S. Cartoon 4), und es gibt Fälle, wo der Text ruhig entfallen könnte, weil er redundant ist, ohne Kommunikationsstörungen herbeizuführen (S. Cartoon 5, 6). Voraussetzung dafür ist, dass das Bild Signale enthält, die zur Bedeutungsdekodierung beitragen, beim Cartoon 5 und 6 ist das die blaue Flagge der Europäischen Union mit (damals noch) 12 gelben Sternen.

Davon ausgehend, dass ein Cartoon aus einem Bild und einem Text besteht, ist angebracht, das einfache Textmodell Autor-Text-Leser zu präzisieren, indem der Autor durch Zeichner und Schreiber ersetzt wird und der Leser durch Beobachter bzw. Zuseher ergänzt wird.

Zwischen Cartoons und erzählten/geschriebenen Witzen lassen sich Ähnlichkeiten und Unterschiede feststellen. Witze werden primär mündlich übertragen, Cartoons dagegen durch Massenmedien. Das bringt Folgendes mit sich: In der unmittelbaren, persönlichen Kommunikation spielt auch Nichtverbales mit, wie Mimik, Gestik, Artikulationsart. Der Kommunikator bestimmt Tempo, Dynamik. In der unpersönlichen, durch Massenmedien verlaufenden Kommunikation kann sich der Leser/Zuschauer dagegen nur darauf verlassen, was als Bild oder Schrift fixiert ist, andere für die Kommunikation wichtige Signale bekommt er nicht, auf der anderen 
Seite bestimmt er selbst das Tempo des Lesens/Zusehens und folglich des Verstehens des Textes, auch kann er auf die Textteile immer wieder zurückkommen.

Bezüglich der textuellen Komponente des Cartoons lassen sich 2 Arten von Texten bestimmen: Kurztexte und Langtexte. Ein Kurztext oder auch Minimaltext stellt das Thema der Textsorte Cartoon dar. Er kommt entweder als Übertitel (S. Cartoon 15) oder als Untertitel (S. Cartoon 5, 6, 7, 8) zum Ausdruck. Das Thema des Textes ist nach Mackelday (Vater, 1992) ein begrifflicher Kern, der aus der Konzentration und Abstraktion des gesamten Textinhalts zu gewinnen ist. Der Minimaltext fungiert somit als eine Art sprachlich konzentrierter/ komprimierter Gebrauchsanweisung zum Verstehen des Textes, als kurzer Kommentar zum Cartoon. Ein Kurztext ist in den meisten Fällen eine Nominalgruppe, Attribute sind auf das Minimum reduziert (Das Europa-Haus, Festung Europa, Festung Europa, Das 19. Loch, Testament). Wo der Text fehlt, muss/ kann er rekonstruiert werden (S. Cartoon 4), wobei dem menschlichen Einfallsreichtum keine Grenzen gesetzt sind. Das Rekonstruieren des Cartoon-Themas kann sich übrigens als eine gute Methode im Fremdsprachenunterricht erweisen. Hier nur zwei Vorschläge zum Cartoon 4: Kantonisierung Bosnien-Herzegowinas, Geburtsstunde des Emmenthalers.

Bei längeren Texten muss das Cartoon-Thema aus dem bildlichen und textuellen Teil des Cartoons erschlossen werden. Es hat den Anschein, dass einzelne Autoren durch die Anhäufung von unnötigen, manchmal sogar von schwer zu enträtselnden Angaben dem Leser das Verstehen des Textes erschweren oder dass sie eine solche für den Witz weitschweifige Textexposition so lange verschleppen, bis der Leser/Zuseher schließlich mit der abschließenden Pointe um so intensiver überrascht wird und damit die Wirkung eines gut erzählten Witzes erzielt ist (S. Cartoon 9). Der Text des Cartoons 9 zeigt, dass die italienischen kulinarischen Einschübe ( $=$ Lachsstreifen an Rucolasalat, Caprese, Pinot Grigio) für die Interpretation des restlichen Textes nicht wesentlich sind, dass darauf also ohne Weiteres hätte verzichtet werden können, doch sie versetzen den Leser in den Zustand des gespannten Erwartens, das schließlich in eine Art für den Witz katartische Entspannung resultiert, die den Höhepunkt der Komik herbeiführt.

Die Struktur des Witzes ist konsequent auf den abschließenden Kommunikationspunkt ausgerichtet, ohne ihn ist die Textsorte nicht existent. Helga Kotthoff (1998) erklärt, was einen guten Witz auszeichnet. Ihre Erklärung lässt sich ohne Weiteres auf Cartoon übertragen:

"Natürlich muss ein guter Witz eine Pointe haben, auf die man niemals selbst gekommen wäre. Es gibt naheliegende Pointen, die den Witz kaputtmachen. Wenn das kommt, was man schon annähernd erwartet hat, ist der Witz strukturell schlecht. Neben der Bedeutung der Pointe ist stets das Witzigste, was für sie eine hohe subjektive Relevanz hat. Empirisch kann ich zeigen, dass das längste Gelächter mit den scherzhaften Bemerkungen erzielt wird, die Bereiche berühren, die für die Lachenden enorm wichtig sind. Abgesehen von der Pointentechnik, die stimmen muss, hat ein guter Witz sehr viel mit subkulturellen Werten und Beobachtungen zu tun, wobei der 
Erzähler eine besonders große Rolle spielt. Auch Schlagfertigkeit ist ein individuelles Talent, das manchen gegeben ist und manchen überhaupt nicht."

In "Witz" (Lixfeld: 1986) ist die Rede von 2 Erzählstrukturtypen des Witzes, mit denen bedingt auch die 2 Erzählstrukturen des Cartoons erklärt werden können.

Der erste Typ ist die zweistufige Erzählstruktur, die meistens aus zwei Sätzen besteht, die einen Minimaldialog konstituieren:

Fragt der Oberkellner: "Wie fanden Sie die Seezunge, mein Herr?"

"Rein zufällig, als ich die Zitronenscheibe zur Seite schob."

Diese Struktur lässt sich z. B. am Cartoon 7 und 8 veranschaulichen, wobei die Funktion des ersten Satzes des Witzes beim Cartoon das Bild übernimmt, die Funktion des zweiten Satzes der Untertitel bzw. der Untertitel und der Text in Sprechblase.

Die dreistufige Erzählstruktur kommt in Einleitung-Steigerung-Pointe zum Ausdruck:

Aus der geschlossenen Abteilung einer Nervenheilanstalt sind während eines Brandes einige Patienten geflohen. Die Wärter machen sich daran, sie wieder einzufangen. (Einleitung)

Als sie nach einigen Stunden wieder zurück sind, fragt der Chefarzt den Oberwärter:

Sind alle zehn ausgebrochenen Patienten wieder da? (Steigerung/Überleitung)

"Was, bloß zehn? Wir haben zwanzig mitgebracht!" (Pointe)

Diese Einleitung-Steigerung-Kulmination-Erzählstruktur kann am Cartoon 10 veranschaulicht werden: Die Funktion der Einleitung übernehmen das Bild und der Texteil: Eine der von Zeus und den Unsterblichen verhängten Qualen des Tantalus dürfte nur wenigen bekannt sein. Die Funktion der Steigerung übernehmen das Bild und der Textteil: Jedesmal, wenn Tantalus sich darüber freuen wollte, unbemerkt aus dem Hof zu treten, wurde er im letzten Moment von der Hausmeisterin Else Rodekirchen angerufen und in ein langes Gespräch verwickelt. Die Funktion der Kulmination übernehmen das Bild und der Textteil: über die in der Hausordnung festgelegten Treppenhausreinigungsbestimmungen, gewisse gesundheitliche Probleme der Frau Rodekirchen und die vorgeblich losen Sitten der jungen Leute im 2. Stock.

Diese Wirkungsmechanismen können die emotionale Reaktion des menschlichen Lachens herbeiführen, wobei wiederum die Lachreaktionen sich in verschiedenen Formen ausdrücken, es ergibt sich eine Palette von Lachformen (Lacharten) wie: inneres Lachen, Lächeln, hämisch lachen, hellauf lachen, freudig lachen, herausprustendes Lachen, höflich lachen, Kichern, von einem Ohr bis zum anderen lachen, Gelächter usw.

Im Grunde genommen lassen sich zwei Hauptfunktionen des Cartoons erkennen: Cartoon ist Träger von Informationen, er schafft oder wirkt auf soziale Denknormen und Verhaltensmuster ein oder sie zumindest widerspiegelt, kritisiert sowohl die 
Gesellschaft als auch das Individuum, er warnt, mahnt, empfiehlt, weist auf Mängel hin, wobei die Karikatur aggressiver oder milder sein kann.

Sigmund Freud hat eine psychoanalytische Witztheorie entwickelt mit Leitbegriffen wie Lustmechanismen, Verdrängung und Tarnung von Aggression, die Beziehung des Witzes zum Traum und zum Unbewussten. Er hat Witze in sogenannte "harmlose" (abstrakte) Witze eingeteilt, deren Lacheffekt allein auf Technik beruht, und auf "tendenziöse", die einer Absicht dienen und bei denen das Lachen auf "Tendenz" zurückgeht.

Indem Helga Kotthoff (1998) die Fragen beantwortet, warum wir so gerne necken und frotzeln, warum Witze erzählt werden und ob der Humor Gruppensolidarität schafft, spricht sie zugleich die zweite Funktion des Witzes an, die auch auf Cartoon übertragen werden kann:

"Ja, das ist eine der wichtigsten Funktionen. Scherzkommunikation schafft Ingroups, wie auch schon Bergson betonte. Andererseits kann sie gleichzeitig auch Outgroups kreieren, wenn wir uns als Deutsche über Schweizer amüsieren oder als Intellektuelle über Manta-Fahrer. Ich kann X necken, der sich wahnsinnig teure Klamotten kauft, indem ich ihn frage, aus welchem Grabbeltisch er seinen Fummel herausgefischt hat, oder ich lade ihn zum Essen ein mit der Begründung, dass es bei ihm angesichts des tollen Outfits zur Finanzierung der Ernährung wohl kaum noch reichen dürfte."

Johan Huizinga (Nusser, 1987: 110) ist ebenfalls der Meinung, dass das Spiel ein Intermezzo im täglichen Leben ist, Betätigung in der Erholungszeit, und zur Erholung dient: "Es schmückt das Leben, es ergänzt es und ist insofern unentbehrlich, unentbehrlich für die Einzelperson als biologische Funktion und unentbehrlich für die Gemeinschaft wegen des Sinnes, der in ihm enthalten ist, wegen seiner Bedeutung, wegen seines Ausdruckswertes und wegen der geistigen und sozialen Verbindungen, die es schafft: kurzum als Kulturfunktion."

Den beiden Cartoon-Typen, d.h. dem gesellschaftskritischen und dem spielerischen l'art pour l'art-Typ liegt Komisches zugrunde. Komik als ästhetische Kategorie ist ein sehr komplexer Begriff, denn sie kommt in verschiedenen Formen zum Ausdruck: Satire, Groteske, Ironie, Zynismus, Possenreisserei, Sarkasmus, Frotzelei, Spott, Blödeln, Parodie usw.

Motivik ist äußerst reich. Konjunktur-Themen sind: Familie, Sex, Gebrechen, soziale Gruppen, Ethnien, Politik, Konfession, Absurdes, Makabres usw., wobei sehr häufig Überschneidungen zu vermerken sind.

Sehen wir uns noch einmal den Cartoon 6 an mit dem Untertitel Festung Europa. Was hat den Autor veranlasst, ihn zu produzieren? Was war sein Ziel? Sieht er die Festung Europa wirklich als ein wackliges Gebäude, das einzustürzen droht? Ist er etwa eingefleischter Befürworter der Idee eines vereinten Europa und gleichzeitig tapfer genug, auch die Vorstellung der Gegenseite zu präsentieren? Oder ist ihm die eine oder die andere politische Idee gleichgültig und will nur provozieren? 
Eine zufriedenstellende Antwort darauf ist nur schwer zu finden, obwohl andernseits doch erschlossen werden kann, was der Autor beabsichtigt hat, vor allem, wenn man seine künstlerische Tätigkeit verfolgt und seine Vorliebe für gewisse Themen kennt. Hat man nur einige seiner Cartoons gesehen, kann man, wenn schon nicht zweifellos sagen, dann wenigstens mit grosser Sicherheit vermuten: ist er dafür, ist er dagegen, ist es ihm gleichgültig, meint er im Ernst, scherzt er nur, frotzelt er etwa, kurz und gut, was sind seine Präferenzen. Am Stil ist der Autor ohne Schwierigkeit zu erkennen (Borislav Sajtinac, Tex Rubinowitz, Bernd Pfarr, Michael Sowa...).

Der Autor bedient sich zahlreicher Techniken zur Erreichung des komischen Effekts. Manchmal macht er in einem Cartoon von verschiedenen Techniken Gebrauch. Die häufigsten sind:

- Personifizierung, Tiere und Sachen werden belebt (S. Cartoon 1, 8, 11, 12),

- Kontrastieren (S. Cartoon 6, 14),

- Banalste und absurdeste Einfälle (S. Cartoon 9, 12, 13, 14),

- Wortspiele (S. Cartoon 2, 3, 15).

In "Witz" (1986: 60, 61) ist die Rolle des Rezipienten des Witzes folgendermaßen beschrieben:

"Die durch den Witz vermittelten, in der Regel auf soziale Konflikte hindeutenden Inhalte werden beim Erzählakt mit den Einstellungen des Rezipienten konfrontiert. Bei diesem Vorgang kann es zu weiteren Konflikten kommen, wenn der vermittelte Inhalt statt auf Zustimmung auf Ablehnung stößt, wenn das erwartete Lachen über den Witz einem betroffenen Schweigen weicht. Aber auch das Lachen kann nicht generell als Befürwortung der inhaltlichen Tendenz eines Witzes angesehen werden. Über einen Witz kann also gelacht werden, ohne dass man sich mit seiner kritischen Aussage identifiziert."

Nicht jeder empfindet den Witz gleich. Was ich für witzig halte, muss nicht witzig für Andere sein und umgekehrt. Es gibt Individuen, die auf bestimmte Witze lebhaft reagieren und andere, die denselben Witz überhaupt nicht komisch finden.

Helga Kotthoff (1998) antwortet auf die Frage, inwiefern sich in der Scherzkommunikation Moralvorstellungen zeigen:

"Moralvorstellungen müssen im weitesten Sinne als eine Unterscheidung zwischen Gut und Böse verstanden werden. Wenn man eine bestimmte Gruppe beobachtet, dann zeigt sich im Lachverhalten deren Wertestruktur. Beispiele zeigen das am besten. Nehmen wir eine Gruppe ökologisch orientierter Leute, so lässt sich bei ihnen zeigen, wie sie sich über den Otto Normalverbraucher sehr gerne mokieren. In einem solchen Milieu würden mit Sicherheit keine Anti-Türkenwitze erzählt, denn niemand würde mitlachen. Dort erzeugen diese enorme Empörung, während in rechtsradikalen Kreisen die Anti-Türkenwitze sich ja leider einer gewissen Beliebtheit erfreuen. Und an sogenannten heiteren Abenden einer Akademikergruppe ließe sich 
beobachten, wie oft hier sehr Triviales gerade in Scherzen ungeheuer aufgewertet wird."

Nun ist es klar, dass der Rezipient beim Witz oder Cartoon (am Ende) das Unerwartete erwartet, sonst hätte er ihn überhaupt nicht gelesen. Nachdem er den Text gelesen hat, kann er ihn verstehen oder eben nicht. Wovon hängt denn das ab? Hier kommt es auf Fähigkeit und Bereitschaft des Rezipienten an. Unter Fähigkeit versteht man schnelle Gedankenverbindung, die Leichtigkeit des Assoziierens, die Fähigkeit, sich in nicht real existierende Welten zu projizieren, Phantasie wird vorausgesetzt. Unter Bereitschaft versteht man die Einstellung zum Thema: Wenn man fest an die Idee eines vereinten Europa glaubt, wird man den Cartoon 6 (Festung Europa) gemein finden. Wenn von Priestern, die in Zölibat leben sollten, Witze erzählt werden, in denen von ihrem Sexualleben die Rede ist, wird das bei ihnen und ihren Anhängern auf Ablehnung stoßen, sie werden sich betroffen fühlen. Das Lachen wird ihnen im Hals steckenbleiben.

Aus den 15 in diesem Beitrag gesammelten Cartoons ist es jedoch zu schließen, dass die Autoren keine ähnlichen Konfliktsituationen beabsichtigt haben. Der Cartoon als eine von komprimiertesten Textformen verlangt relativ viel intellektuelle Anstrengung sowohl von Seiten des Produzenten als auch des Rezipienten. Je unwahrscheinlicher, verspielter, realitätsferner, konstruierter, absurder, banaler die Inhalte sind, desto mehr scheinen sich die beiden Seiten daran zu erfreuen. Die Versuchung, sich zu Exzentrik neigende Situationen auszudenken und sie zu verstehen, ist für den Produzenten und Rezipienten zu gross, als daß sie ihr widerstehen könnten.

Cartoon 1

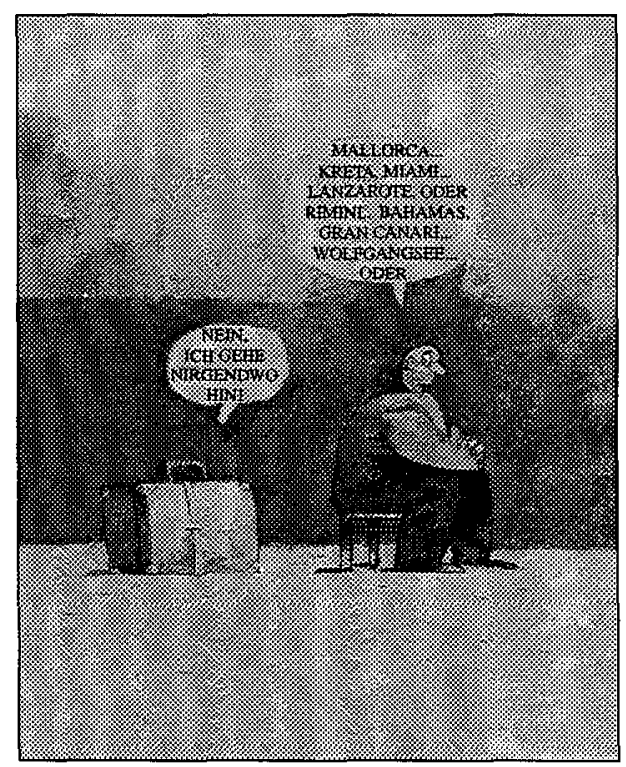

\section{Cartoon 2}

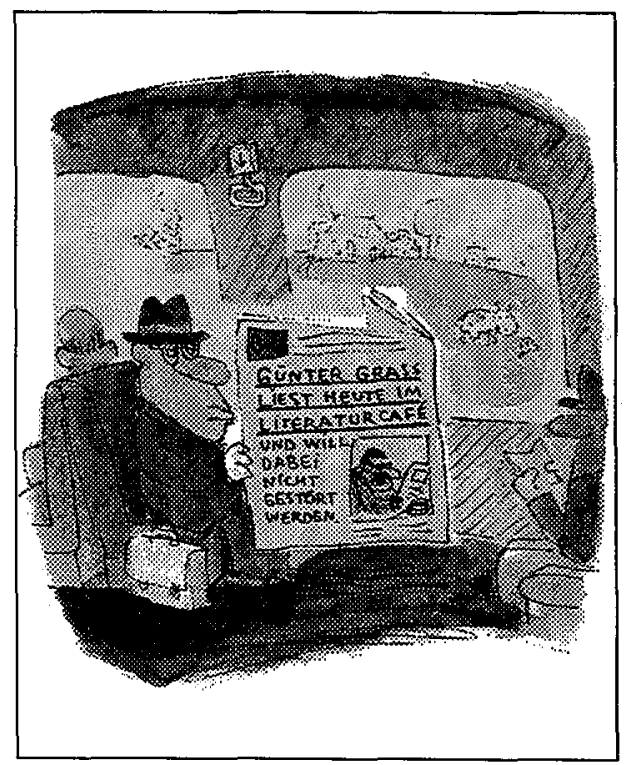


Cartoon 3

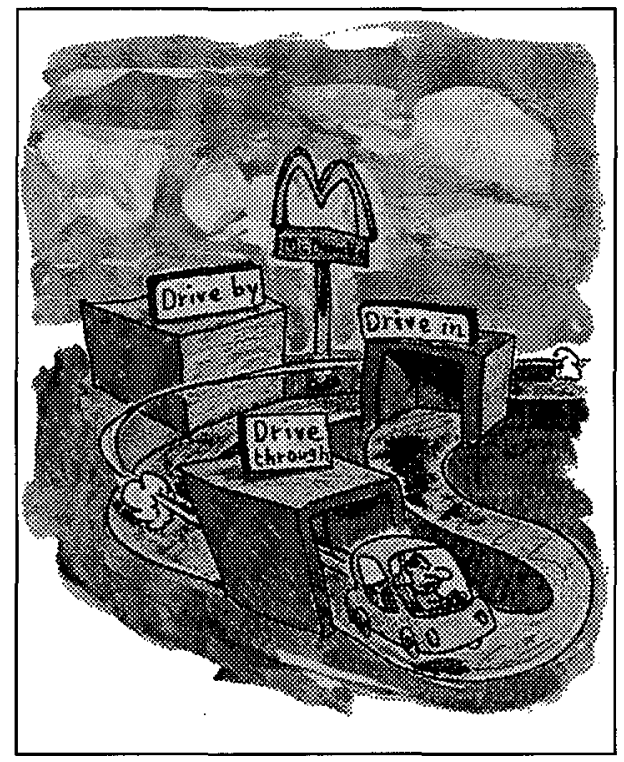

Cartoon 5

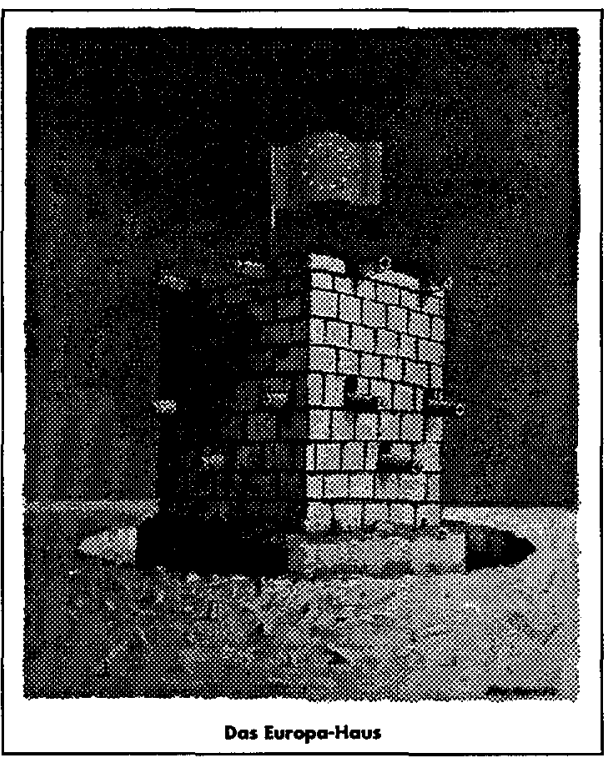

\section{Cartoon 4}

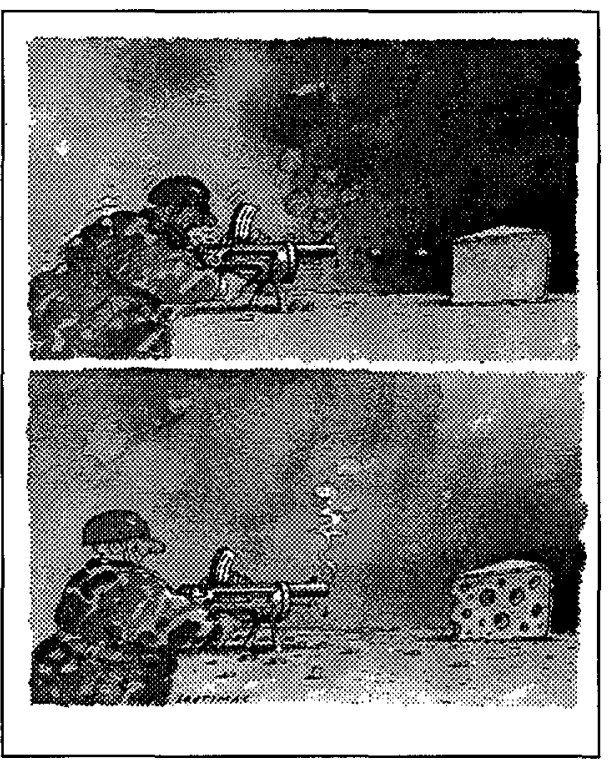

Cartoon 6

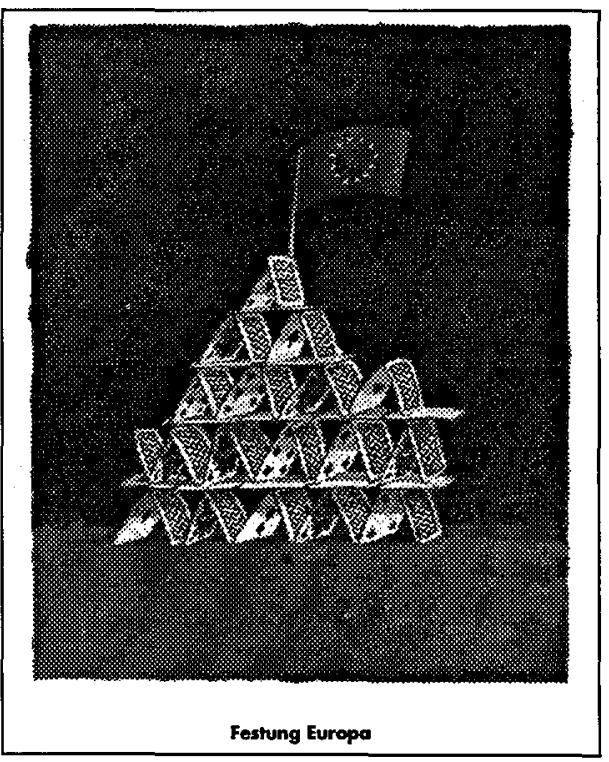


Cartoon 7

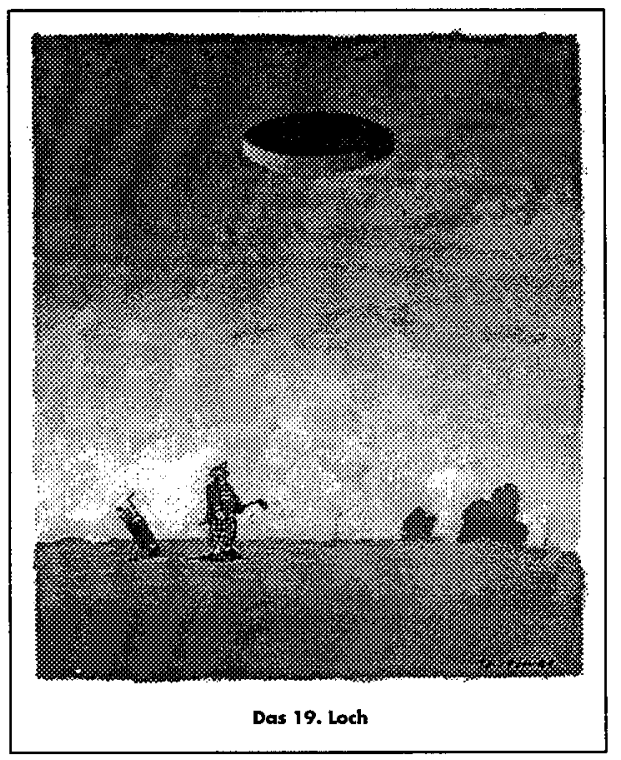

\section{Cartoon 8}

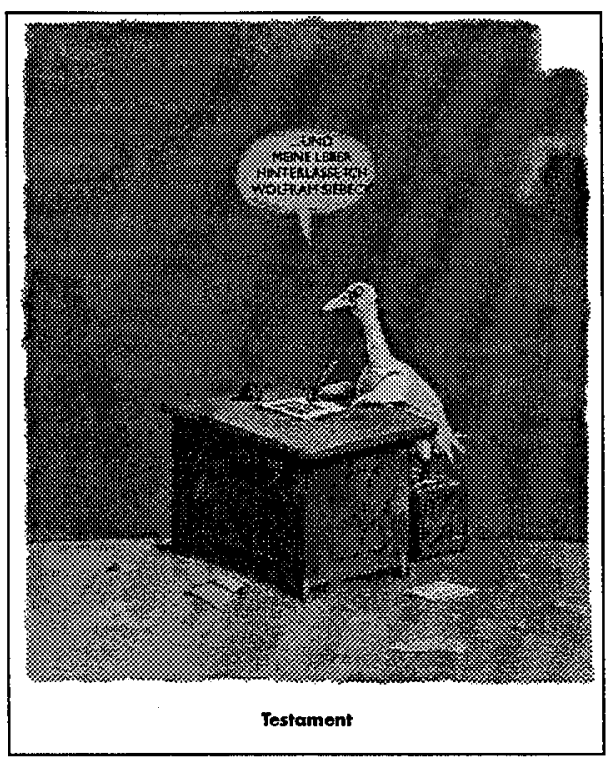




\section{Cartoon 9}

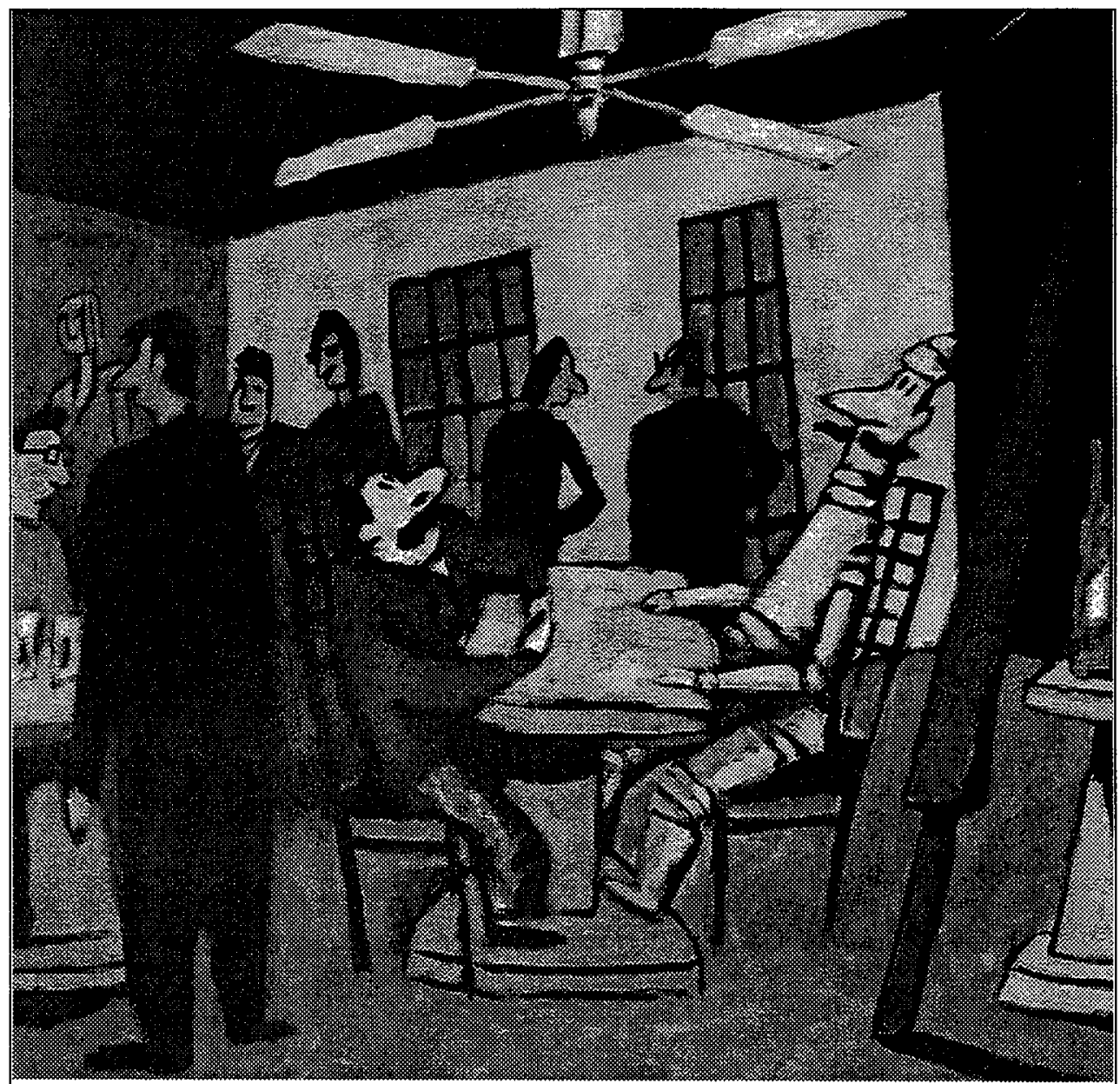

Don Gulchotte hatte gerade filr

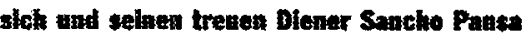
in der Szenthmelpe "Caft $\mathrm{X}_{\text {s }}$

in aller Rabe whe Portion Lachestrelfon an Aucolasalat, einen Caprese and zwei clas Pinot Grigio bestelit, da thed sein Bllek asf dea Ventilator $\ldots$. . 


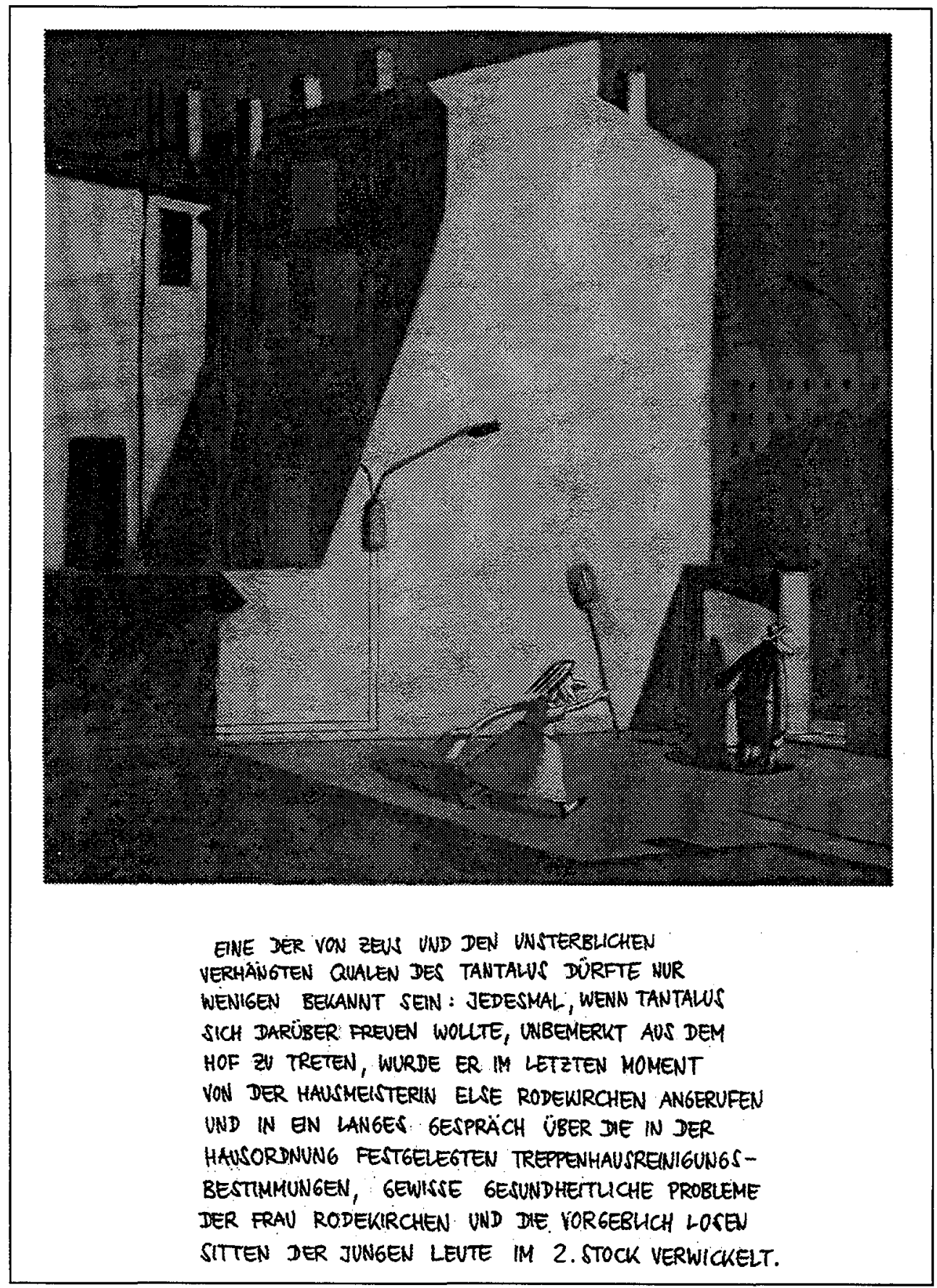




\section{Cartoon 11}

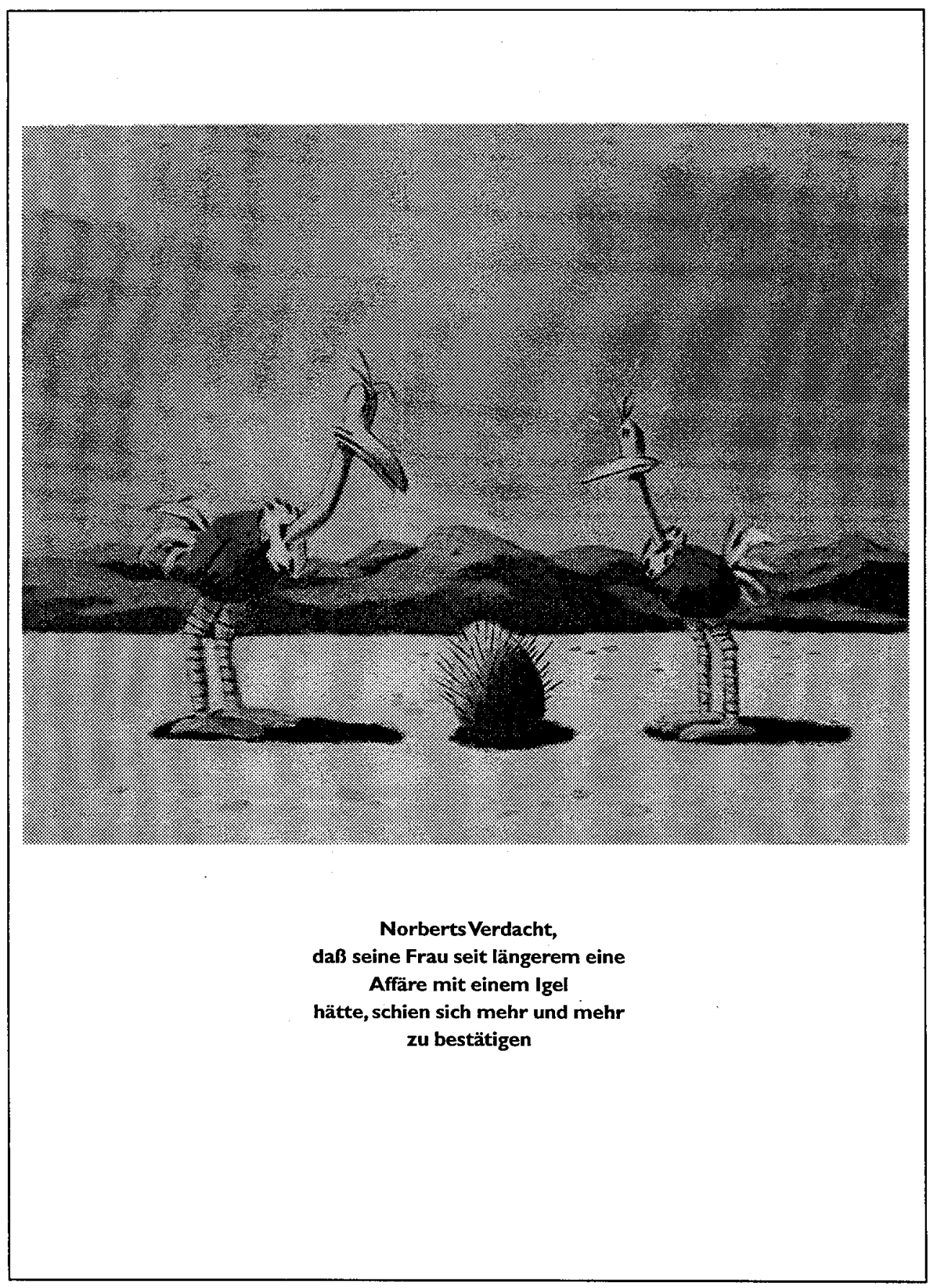




\section{Cartoon 12}

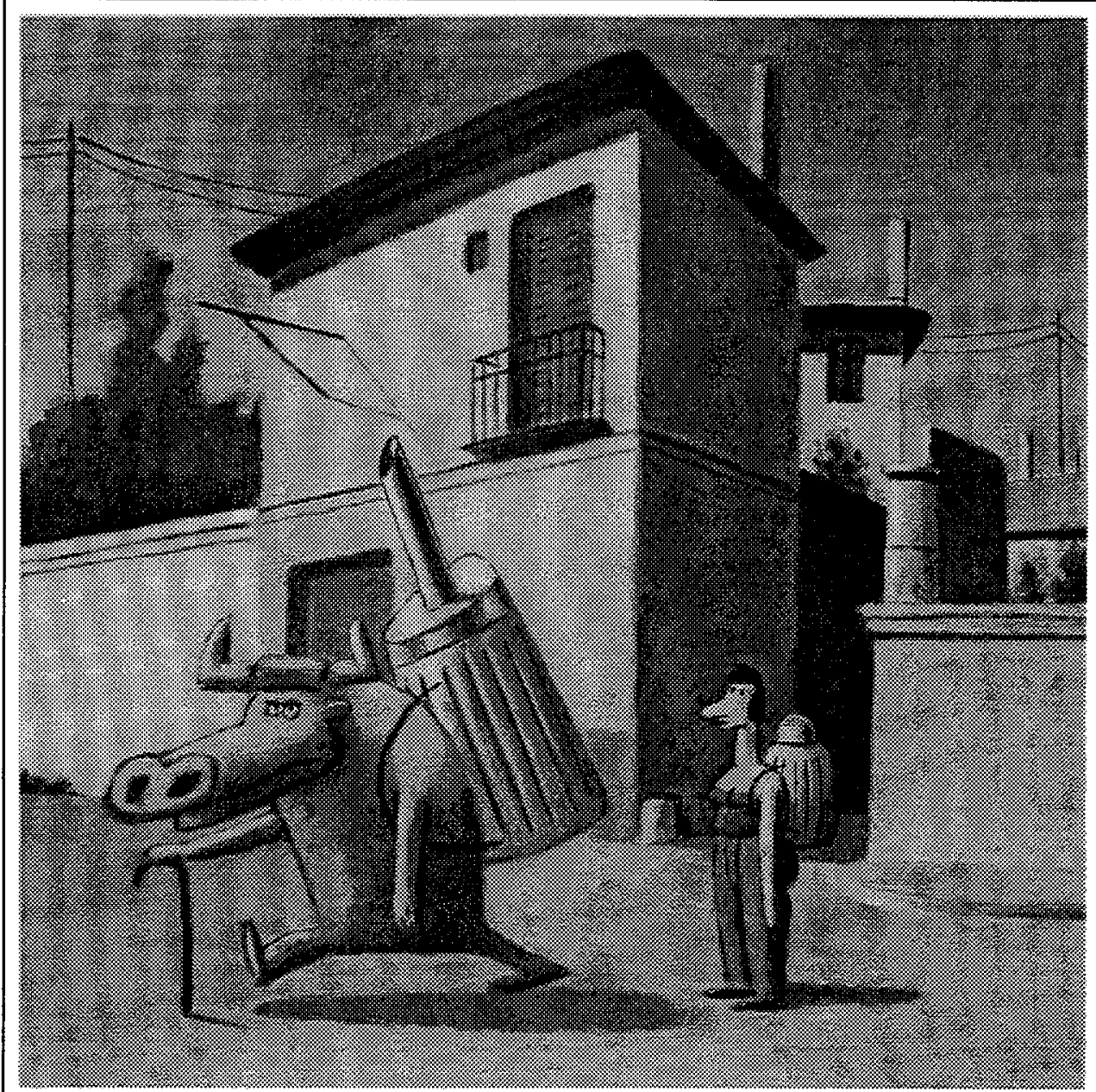

Für die modebewubte Gerda war der Spanientrip ziemlich frustrierend:

Selbst alte, ausgediente

Kampfstiere hatten eindrucksvollere

Rucksäcke ats sie 


\section{Cartoon 13}

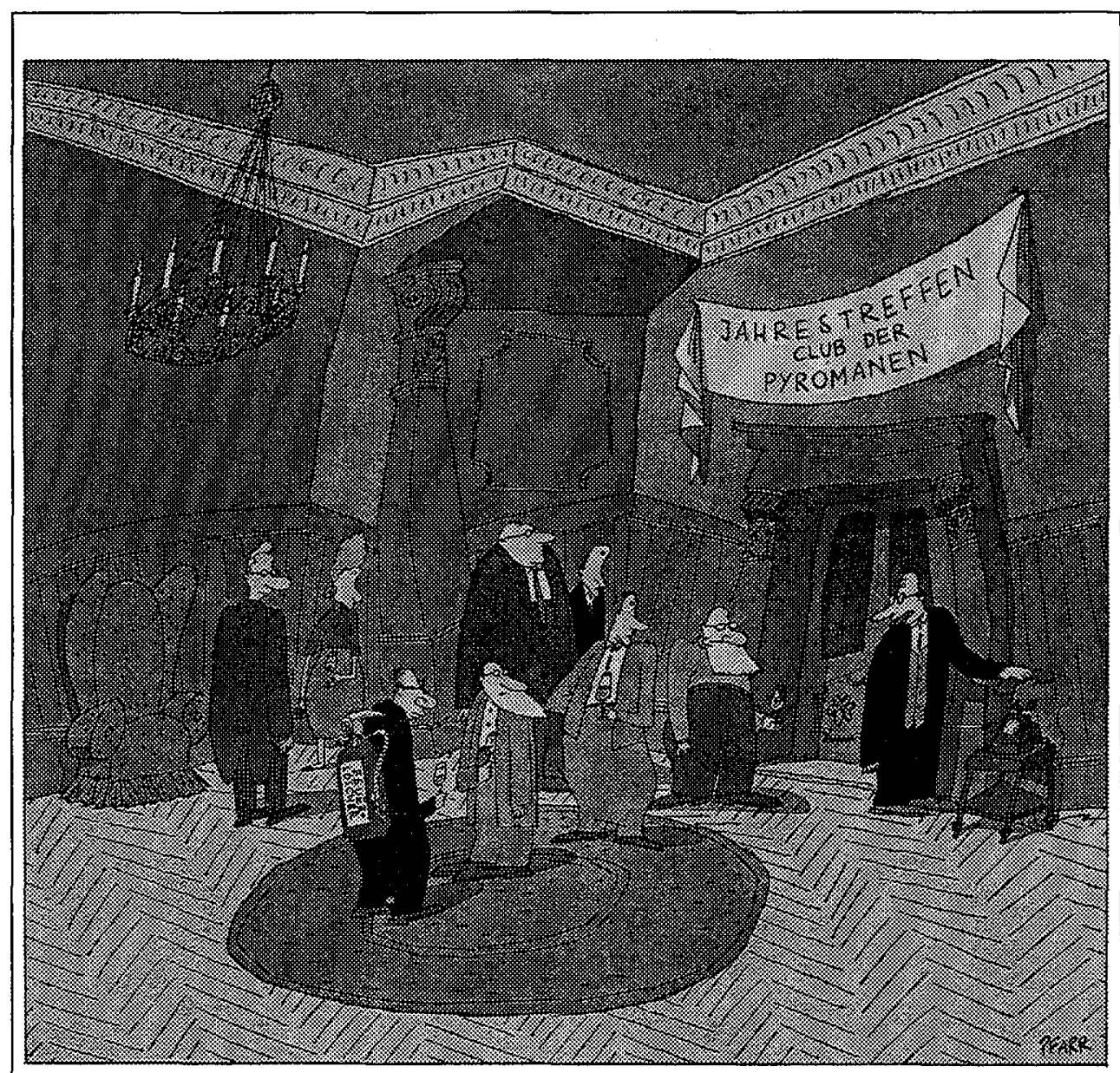

* Soeben habe ich die

Nachricht emalten, dab sich unter uns ein Betrager befladetik 
Cartoon 14

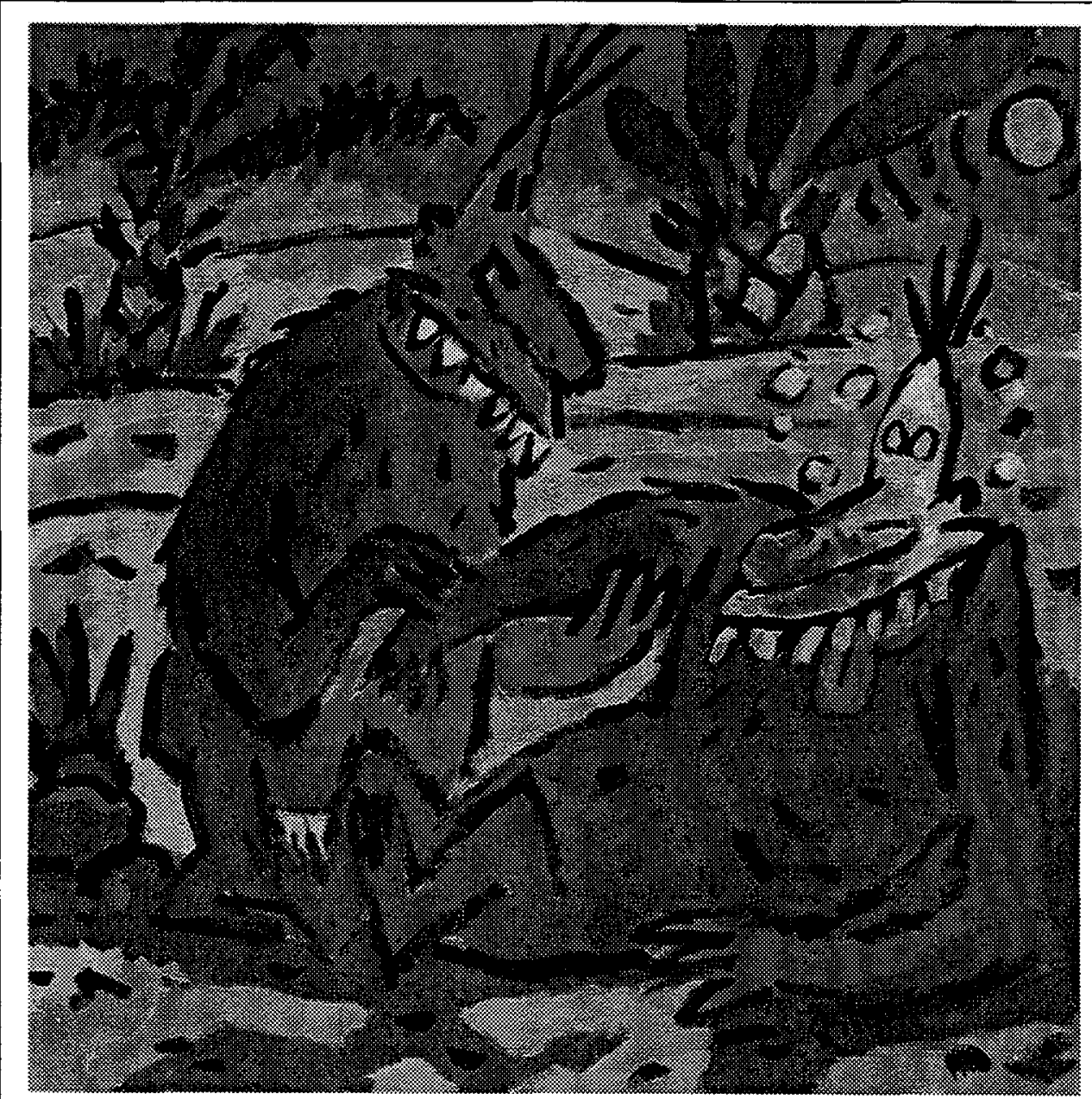

Als der Yeti, der abscheulliche Schneemensch, seinen Vetter, den abscheulichen Sandmenschen, in

dessen Heimat, der Sahara, besuchte, hatte er leider vom ersten Tag an enorme Kreislaufprobleme wegen der Hitze 


\section{Cartoon 15}

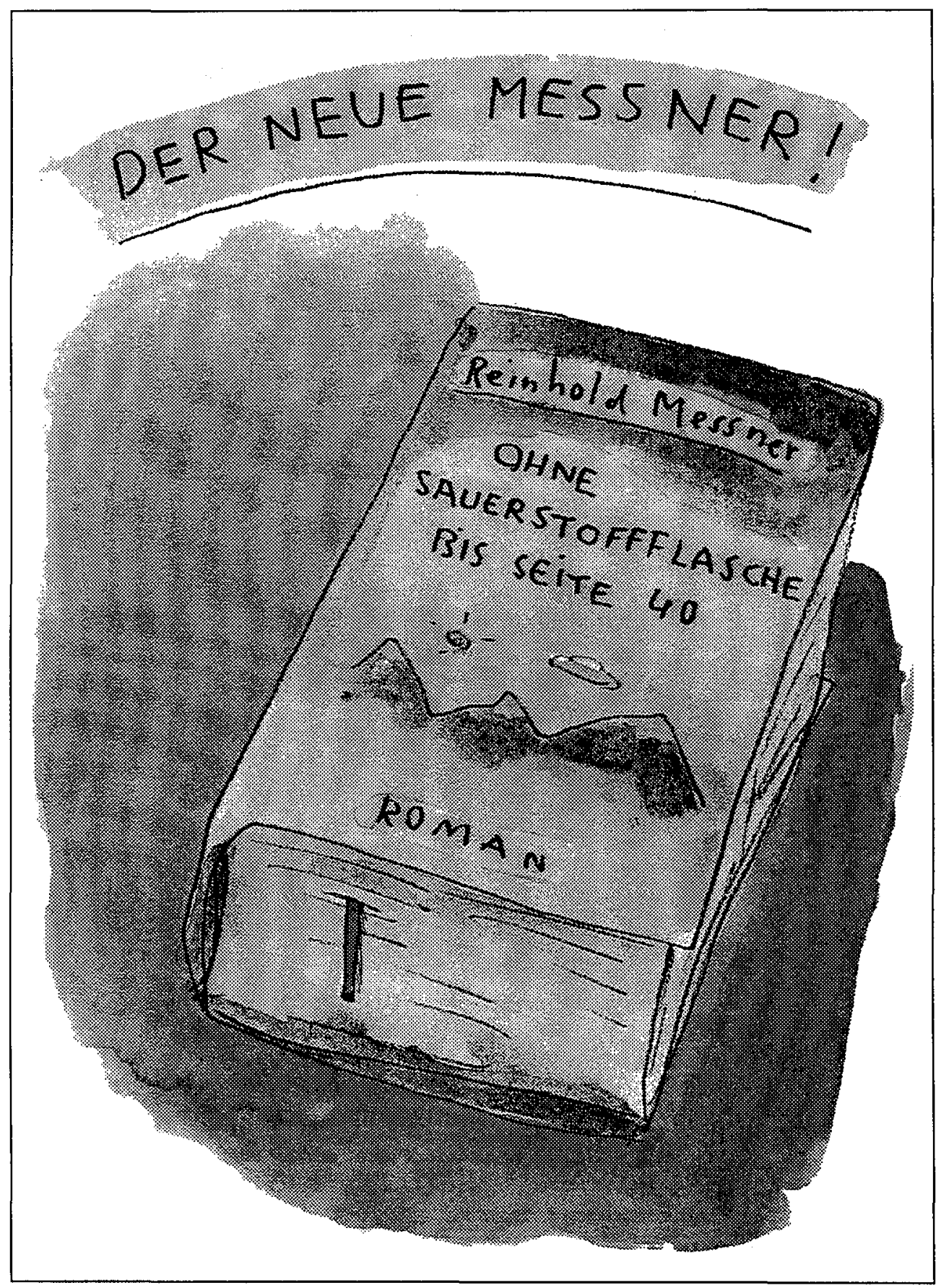




\title{
Zusammenfassung
}

Gegenstand dieses Beitrags ist die Textsorte Cartoon in "Zeitmagazin". Der Cartoon besteht aus dem bildlichen und dem textuellen Teil. Der textuelle Teil wird in Form von Kurz- oder Langtext realisiert. Es lassen sich strukturelle Ähnlichkeiten zwischen Cartoons und Witzen beobachten. Gut strukturierte Witze und Cartoons können die emotionale Reaktion herbeiführen, die in verschiedensten Lachreaktionen zum Ausdruck kommt. Der Cartoon als kulturelle Kommunikation übt die gesellschaftskritische und die spielerische Funktion (Spiel für Erwachsene) aus. Die beliebtesten Techniken zur Erreichung des komischen Effekts sind Personifizierung von Tieren und Sachen, Kontrastieren, Gebrauch von banalsten und absurdesten Einfällen, Wortspiele. Der Rezipient kann die Mitteilung verstehen oder nicht, er kann ihr zustimmen oder sie ablehnen. Je einfallsreicher, realitätsferner, absurder in ihrer Banalität die Inhalte der Cartoons sind, desto intensiver scheint die künstlerische Perzeption des Rezipienten zu sein.

\section{Literatur}

Bergson, Henri (1972): Das Lachen. Zürich

Eco, Umberto (1991): Einführung in die Semiotik. Autorisierte deutsche Ausgabe von Jürgen Trabant. München.

Kotthoff, Helga (1998): Warum lachen Frauen anders, Frau Kotthoff (Ein Interview von

Dieter Wöhrle) In: Frankfurter Allgemeine Magazin, 13.2.1998, 50-51.

Lixfeld, Hannjost (Hrsg.) (1986): Witz. Stuttgart..

Marfurt, Bernhard (1977): Textsorte Witz. Möglichkeiten einer sprachwissenschaftlichen Textsorten-Bestimmung. Linguistische Arbeiten 52. Tübingen.

Nusser, Peter (Hrsg.) (1987): Schwarzer Humor. Stuttgart.

Vater, Heinz (1992): Einführung in die Textlinguistik. München.

Weber, Heinz (1984): Elements of Text-Based and Image-Based Connectedness in Comic Stories, and Some Analogies to Cinema and Written Text. In: Text and Discourse Connectedness. Proceedings of the Conference on Connexity and Coherence, Urbino, July 16-21, 1984. Urbino.

\author{
Povzetek \\ O NASTAJANJU IN RECEPCIJI KARIKATURE V "ZEITMAGAZIN-U"
}

\footnotetext{
Predmet tega prispevka je besedilna vrsta karikatura v Zeitmagazinu. Karikaturo sestavljata slikovni in jezikovni del. Jezikovni del je kratek ali dolg. Med karikaturo in šalo je opaziti strukturne podobnosti. Dobro zgrajene karikature in šale lahko sprožijo čustveno reakcijo, ki se kaže v široki paleti smeha. Karikatura kot kulturna komunikacija ima dve funkciji: lahko je družbena kritika ali zgolj igra za odrasle. Priljubljena sredstva za doseganje komičnega učinka so poosebljanje živali in stvari, kontrastiranje, raba banalnih in absurdnih domislic, besedne igre. Bralec/gledalec sporočilo razume ali ne, lahko se z njim strinja, lahko ga tudi zavrača. Bolj ko je vsebina karikature domiselna, nerealistična, v svoji banalnosti absurdna, toliko intenzivneje jo doživlja bralec oz. gledalec.
} 